\title{
CAZA Y ESPACIOS NATURALES PROTEGIDOS EN EXTREMADURA
}

\author{
Juan Ignacio Rengifo Gallego \\ Departamento de Arte y Ciencias del Territorio. Universidad de Extremadura \\ irengifo@unex.es \\ José Manuel Sánchez Martín \\ Departamento de Arte y Ciencias del Territorio. Universidad de Extremadura \\ jmsanche@unex.es
}

\section{RESUMEN}

La superficie protegida ha crecido, en poco más de un siglo, de forma muy rápida, logrando alcanzar cifras significativas que, no obstante, pueden oscilar de forma muy notable entre unos países y otros. Este interés por proteger los espacios naturales responde, en el contexto actual, a la consecución de una amplia serie de fines de carácter científico, educativo, recreativo y socioeconómico. Como consecuencia de ello, ha surgido un nuevo escenario en el que determinados aprovechamientos tradicionales se están viendo afectados.

La caza es, sin duda, uno de los mejores ejemplos de actividad tradicional que se está viendo perjudicada por este proceso. Con estos antecedentes, en el presente trabajo se analiza con carácter general la situación de los espacios naturales protegidos en Extremadura en relación con la actividad cinegética. Este análisis se lleva a cabo teniendo en cuenta que un tercio de la superficie regional está protegida por algunas de las figuras existentes y que, además, estos espacios se distribuyen por áreas en las que la caza ha sido un aprovechamiento tradicional que ha contribuido a mantener los espacios en mejor estado de conservación.

Palabras clave: caza; espacios protegidos; Extremadura; nuevo escenario.

\section{ABSTRACT}

\section{Hunting and protected natural areas in Extremadura}

Protected areas have grown very quickly in the last century, achieving significant figures which, however, vary very significantly from one country to another. This interest in protecting natural areas is related, in the current context, with different objectives: scientific, educational, recreational and socioeconomic. As a result, we have a new scenario in which certain traditional uses are being affected.

Hunting is undoubtedly one of the best examples of traditional activity that is being affected by this process. With this background, this paper analyses the general situation of protected natural spaces in Extremadura in relation to hunting. This analysis takes into account the fact that a third of the regional territory is protected by some of the existing mechanisms and, moreover, these spaces are distributed in areas where hunting has been a traditional activity that has helped to keep the spaces in a good state of conservation.

Keywords: Hunting; protected areas; Extremadura; new scenario.

\section{INTRODUCCIÓN}

La historia de la protección de los espacios naturales protegidos, tal y como la entendemos hoy, es corta pero intensa. Con la recurrentemente citada declaración de Yellowstone como Parque Nacional en Estados Unidos, acontecimiento que se produjo en el último cuarto del siglo XIX, se inició una nueva 
era que, tras no pocas vicisitudes, se ha prolongado hasta nuestros días. Esta etapa, de algo más de un siglo de duración, se ha caracterizado, por una parte, por la constante agregación de superficie protegida y la paulatina mundialización de las políticas proteccionistas y, por otra, por el desarrollo de un corpus normativo cada vez más complejo. En realidad, este periodo puede parcelarse en diferentes fases que se pueden concretar cronológicamente, de acuerdo con lo expuesto por Tolón Becerra y Lastra Bravo (2008), en tres: primera fase, con una extensión temporal que se extiende entre 1872-1975, y que se caracteriza por el "nacimiento, organización y consolidación de los primeros Espacios Naturales Protegidos, principalmente Parques Nacionales"; segunda fase, determinada por el incremento de los Espacios Naturales Protegidos, tanto desde el punto de vista numérico como superficial y, por último, una tercera fase, que se desarrolla a partir de la celebración de la Cumbre de Río en 1992, que incorpora una nueva concepción que vincula la protección de los espacios con los principios asociados a la sostenibilidad. El resultado final de este proceso se ha saldado, por un lado, con una superficie terrestre protegida a escala planetaria que actualmente se estima en el $14,6 \%$, a la que habría que añadir un $9,7 \%$ de aguas costeras (Europarc, 2014) y, por otro, con el desarrollo de un concepto de conservación ligado a los espacios, mucho más complejo e integrador que en sus inicios, en el que se combinan diferentes fines de carácter científico, educativo, recreativo y socioeconómico. Mientras tanto, hay que señalar que este proceso de crecimiento se ha desarrollado en un entorno que se ha caracterizado por una creciente e imparable internacionalización económica, la constante construcción de todo tipo de infraestructuras y equipamientos que se vio acompañado, asimismo, de un incremento sostenido de la población absoluta mundial, que superó en el año 2013 el umbral de los 7.200 millones de habitantes.

Dicha deriva global se vio reflejada en Europa con un aumento del porcentaje de la superficie protegida hasta alcanzar proporciones muy significativas, especialmente tras el desarrollo de la Red Natura 2000, circunstancia que ha permitido a los países miembros de la UE contar con una red ecológica paneuropea de una extensión considerable en un periodo de tiempo muy corto. La Red Natura 2000, nacida de la Directiva Hábitats del año 1992, está formada por las Zonas de Especial Protección de Aves (ZEPAS), figura existente desde la promulgación de la Directiva Aves del año 1979, y los Lugares de Importancia Comunitaria (LIC) hasta su transformación en Zonas de Especial Conservación (ZEC). Como resultado de esta política, la Red Natura 2000 cuenta en Europa con más de 750.000 km², siendo España la nación que aporta la mayor extensión de superficie protegida a esta red: $137.000 \mathrm{~km}^{2}$ ( $17 \%$ del total europeo). Tras España se posicionan países como Francia con $68.000 \mathrm{~km}^{2}$ o Polonia con $60,700 \mathrm{~km}^{2}$ (datos del año 2010, disponibles en http://ec.europa.eu/).

Por último, y en lo que se refiere a España en el contexto actual, país que cuenta con una historia ligada a la conservación de los espacios que se remonta al año 1916, tras la aprobación de una Ley de Parques Nacionales que, en términos generales, puede considerarse pionera, la superficie total protegida alcanza el 27\% (año 2011). Por su parte, si se toma como referencia lo que ocurre en las comunidades autónomas se constatan las diferencias porcentuales entre los distintos entes territoriales, que oscilan entre el 22,74\% del País Vasco y el 74,58\% de Canarias. Estas cifras son el resultado de la suma de las superficies con las que cuentan las diferentes figuras integradas en la Red de Espacios Naturales Protegidos (ENP), Red Natura 2000 y los Instrumentos Internacionales, de acuerdo con las cifras expuestas en cualquier inventario oficial (Magrama, 2013).

Tal y como se ha expuesto de forma sintética, el crecimiento de la superficie protegida ha experimentado en el periodo más reciente de la edad contemporánea, independientemente de la escala geográfica tenida en cuenta, un incremento muy notable. Como consecuencia de ello, partimos de la hipótesis de que en estos terrenos se han generado nuevas condiciones en lo que a usos y aprovechamientos tradicionales y emergentes se refiere, siendo la caza una de las actividades afectadas. Con estos antecedentes, el objetivo de este artículo se ajusta a la realización de un análisis sobre la actividad cinegética en los espacios naturales protegidos de Extremadura, en un contexto complejo que se define por la primacía de cuatro factores que se interrelacionan entre sí:

- Crecimiento exponencial de la superficie protegida en Extremadura desde el año 1979.

- Continuidad de la práctica de la actividad cinegética en Extremadura en dos direcciones (deportiva y económica) con lo que ello conlleva en términos espaciales, sociales y económicos.

- Florecimiento de nuevas actividades de ocio en los espacios naturales.

- Desarrollo de su corpus normativo. 
El artículo se estructura, en coherencia con los cuatro aspectos señalados anteriormente, en sendos bloques donde se aborda, en primer lugar, el crecimiento de los espacios naturales protegidos en Extremadura desde la fecha en la que se declaró el primer espacio en la Comunidad Autónoma. Posteriormente, se analiza el peso que la actividad cinegética sigue teniendo en la actualidad en el territorio extremeño y cómo tiene que compartir sus espacios tradicionales con otros aprovechamientos de tipo recreativo. Finalmente, se hace un repaso del complejo corpus normativo desarrollado por la comunidad autónoma, prestándole especial atención al rol que juegan las diferentes herramientas de gestión y planificación, como resultado del papel que éstas tienen a la hora de expresar los usos y aprovechamientos compatibles con la conservación.

\section{LOS ESPACIOS NATURALES PROTEGIDOS DE EXTREMADURA}

Diversos factores de índole histórico, social y económico han favorecido que Extremadura sea una comunidad autónoma con un sobresaliente nivel de conservación de la naturaleza. Sin embargo, no fue hasta el año 1979 el momento en el que se declaró a Monfragüe (17.852 hectáreas) como Parque Natural, siendo el primer espacio natural protegido a nivel regional. Dicha declaración se realizó al amparo de la Ley 15/1975 de Espacios Naturales Protegidos (BOE núm. 107, de 5 de mayo de 1975) en la que se definieron una serie de figuras que vinieron a enriquecer a las existentes con carácter previo: la de parque nacional, desde el año 1916, y la de monumento natural de interés nacional y la de sitio natural de interés nacional, desde el año 1927. En la Ley 15/1975 las nuevas figuras creadas fueron las reservas integrales de interés científico, parques naturales y parajes naturales de interés nacional. Posteriormente, con la llegada de la democracia, vendría el Estado de las Autonomías y la posibilidad de que éstas asumieran "la gestión en materia de protección del medio ambiente" (Artículo 148 de la Constitución Española), tal y como recogieron sucesivamente los diferentes Estatutos de Autonomía aprobados en sedes parlamentarias regionales.

Asimismo, fue fundamental para el crecimiento de la red de espacios protegidos en España la aprobación, casi tres lustros más tarde, de la Ley 4/89 de Conservación de la Naturaleza y de la Flora y Fauna Silvestres, que derogó a la anterior de 1975, en la que se contempló un mayor número de figuras protectoras: parques, reservas naturales, monumentos naturales y paisajes protegidos. Del mismo modo, en dicha Ley se abría la posibilidad de que las comunidades autónomas pudieran establecer, además de las figuras reseñadas, otras diferentes, como así hicieron en años posteriores. En la Comunidad Autónoma de Extremadura esta posibilidad se plasmó, tras la pertinente sanción en la Asamblea de Extremadura, en la Ley 8/1998, de 26 de junio, de Conservación de la Naturaleza y de Espacios Naturales de Extremadura. En dicha norma se recogieron hasta 10 figuras diferentes: parque natural, reserva natural, monumento natural, paisaje protegido, zona de interés regional, corredor ecológico y de biodiversidad, parque periurbano de conservación y ocio, lugar de interés científico, corredor ecocultural y árbol singular.

Por su parte, mientras el corpus normativo medioambiental iba desarrollándose, tras la sucesiva aprobación de normas promulgadas a escala regional, nacional y europea, la red de espacios protegidos de Extremadura iba creciendo, tal y como se expresa a continuación, conforme a la siguiente secuenciación diacrónica:

- Década de los setenta. Fue el punto de partida tras la aprobación del primer espacio natural protegido en Extremadura a finales de la década de los setenta (1979): Parque Natural de Monfragüe con 17.852 hectáreas.

- Década de los ochenta. Durante la década de los ochenta se produjo un acontecimiento de especial importancia para España, como fue la incorporación de nuestro país a la Unión Europea. Dicha incorporación tuvo importantes consecuencias en términos ambientales al ser factible la posibilidad de aprobar la protección de espacios, conforme a la figura conocida como Zona de Especial Protección de Aves (ZEPA), vigente desde el año 1979, fecha en que se publicó la Directiva Aves. Como resultado de ello, en el año 1989 se declararon las primeras ZEPAs de Extremadura, incrementándose sustancialmente el territorio protegido a escala regional tras la incorporación a su incipiente red de las siguientes ZEPAs: Sierra de San Pedro, Llanos de Cáceres-Sierra de Fuentes, Sierra de Hornachos, Embalse de Orellana-Sierra de Pela, Monfragüe y Embalse de Cornalvo.

- Década de los noventa. Durante la década de los noventa se produjo la aprobación de dos hitos normativos que jugarían un papel decisivo en el crecimiento de los espacios protegidos de la región, 
especialmente, durante la primera década del siglo XXI. El primero de ellos fue la aprobación a escala europea de la Directiva Hábitat en el año 1992, bajo cuyo paraguas nació la Red Natura 2000, en la que se incluyeron las figuras constituidas por los LIC (hasta su conversión en ZEC) y las ya creadas ZEPAs. En cuanto al segundo de los hitos normativos, consistió en la aprobación de la ya citada primera Ley regional de Espacios Naturales Protegidos durante el año 1998, en la que se incluyeron diez figuras distintas. Inequívocamente, tras la publicación de esta norma, la red de espacios naturales protegidos adquirió una nueva dimensión, no solo por el establecimiento de nuevas figuras de protección, sino por el desarrollo complementario de los instrumentos de ordenación, gestión y el régimen jurídico (Fernández, 2005). De cualquier forma, y al margen de la aprobación de las normas antes mencionadas, la superficie protegida creció de forma tímida en esta década tras la incorporación de una serie de nuevos espacios protegidos de escasa entidad territorial: Reserva Natural de la Garganta de los Infiernos (1994), Monumento Natural de Cuevas del Castañar (1997), Monumento Natural de Los Barruecos (1996) y Monumento Natural Mina de la Jayona (1997).

- Primera década del siglo XXI. Con los antecedentes de las figuras creadas por la Ley regional de espacios protegidos de 1998, dio comienzo una etapa muy fructífera desde el punto de vista de la aprobación de una extensa nómina de ENP, tal y como se aprecia en la Tabla 1, incluyendo un nuevo Parque Natural: Parque Natural del Tajo Internacional. Esta relación se vio acrecentada por la masiva aprobación, en diferentes años (2000-2003-2004), de una amplia nómina de espacios pertenecientes a la Red Natura 2000 (ZEPAs y LICs), hasta que la Comisión Europea consideró en el año 2006 que las 69 ZEPAS gozaban de la suficiente entidad superficial y representatividad. Finalmente, durante el año 2012, con el objeto de "mejorar la coherencia de la Red y teniendo en cuenta la nueva información disponible se procedió a la revisión y actualización de los límites de los lugares Natura 2000 en Extremadura declarados hasta la fecha, y a la propuesta de dos nuevas ZEPA y dos nuevos LIC" (Decreto 110/2015, por el que se regula red ecológica europea natura 2000 en Extremadura).

Las nuevas declaraciones trajeron diferentes consecuencias que se pueden concretar en el paulatino incremento de la superficie protegida que, en la actualidad, se sitúa en el 30,61\% de la superficie regional. A este porcentaje no se hubiera llegado sin el aporte superficial de las figuras que componen la Red Natura 2000, habida cuenta de que los ENP solo afectan al 7,5\% de la superficie regional. En la actualidad (2015), la Red Natura 2000 está integrada por 160 espacios que se distribuyen por 1.264.288 hectáreas, el 30,3\% de la superficie regional (Tabla 2).

Tabla 1. Fecha de declaración de los ENP de Extremadura en el periodo 1979-2010

\begin{tabular}{|c|c|}
\hline NOMBRE DEL ESPACIO PROTEGIDO & Año de declaración \\
\hline \multicolumn{2}{|l|}{ ANTERIORES A LA LEY 8/1998 } \\
\hline Parque Natural de Monfragüe & 1979 \\
\hline Reserva Natural de la Garganta de los Infiernos & 1994 \\
\hline Monumento Natural de Los Barruecos & 1996 \\
\hline Monumento Natural de La Jayona & 1997 \\
\hline Monumento Natural de Cuevas de Castañar & 1997 \\
\hline \multicolumn{2}{|l|}{ POSTERIORES A LA LEY 8/1998 } \\
\hline Monumento Natural Cuevas de Fuentes de León & 2001 \\
\hline Parque Periurbano de Conservación y Ocio Moheda Alta & 2001 \\
\hline Parque Periurbano de Conservación y Ocio La Sierra & 2002 \\
\hline Lugar de Interés Científico Volcán el Gasco & 2003 \\
\hline Corredor Ecológico y de Biodiversidad Pinares del Tiétar & 2003 \\
\hline Corredor Ecológico y de Biodiversidad Río Guadalupejo & 2003 \\
\hline Corredor Ecológico y de Biodiversidad Río Bembézar & 2004 \\
\hline Parque Natural de Cornalvo & 2004 \\
\hline Paisaje Protegido Monte de Valcorchero & 2005 \\
\hline Parque Periurbano de Conservación y Ocio La Charca de Brozas y Ejido & 2005 \\
\hline
\end{tabular}




\begin{tabular}{|l|c|}
\hline Parque Periurbano de Conservación y Ocio La Pisá del Caballo & 2005 \\
\hline Corredor Ecológico y de Biodiversidad Río Alcarrache & 2006 \\
\hline Zona de Interés Regional Sierra de San Pedro & 2006 \\
\hline Zona de Interés Regional Llanos de Cáceres y Sierra de Fuentes & 2006 \\
\hline Zona de Interés Regional Orellana y Sierra de Pela & 2006 \\
\hline Zona de Interés Regional Sierra Grande Hornachos & 2006 \\
\hline Parque Natural del Tajo Internacional & 2007 \\
\hline Árboles Singulares & $\begin{array}{c}\text { Diferentes fechas } \\
\text { posteriores a 2001 }\end{array}$ \\
\hline
\end{tabular}

Fuente: Boletín Oficial del Estado y Diario Oficial de Extremadura. Elaboración propia.

La relación de los espacios incluidos en la Tabla 1 ha variado desde el año 2011, tras la incorporación de nuevos espacios que no han incrementado sustancialmente la superficie protegida. Los cambios que la red ha experimentado se han circunscrito a la aprobación de un nuevo Paisaje Protegido (Castañar Gallego en Hervás), dos Parques Periurbanos de Conservación y Ocio (Dehesa Camadilla en Almaraz y Dehesa Boyal en Montehermoso) y siete nuevos Lugares de Interés Científico.

Tabla 2. Superficie de las áreas protegidas de Extremadura.

\begin{tabular}{|l|c|c|}
\hline & Hectáreas & $\begin{array}{c}\text { Porcentaje superficial } \\
\text { de Extremadura }\end{array}$ \\
\hline ZEPAS (Zona Especial Protección Aves) & 1.102 .741 & $26,5 \%$ \\
\hline ZEC (Zonas de especial Conservación) & 934.118 & $22,4 \%$ \\
\hline ENP (Espacios naturales protegidos) & 314.110 & $7,5 \%$ \\
\hline Red Natura 2000 & 1.264 .288 & $30,2 \%$ \\
\hline Áreas Protegidas (Natura 2000 + ENP) & 1.276 .288 & $30,6 \%$ \\
\hline EXTREMADURA & 4.168 .021 & $100,0 \%$ \\
\hline
\end{tabular}

Fuente: Junta de Extremadura.

\subsection{La era de la planificación}

Por último, la declaración de espacios tuvo otros efectos como la necesidad de diseñar y aprobar diferentes instrumentos de planificación, gestión, manejo y ordenación, en consonancia con lo dictado por la normativa. En este sentido, la normativa autonómica especifica que los planes de ordenación de los recursos naturales son los instrumentos de planificación y ordenación. A su vez, se definen diferentes instrumentos de manejo y gestión en relación con las diferentes figuras de protección: planes rectores de uso y gestión (parque natural, reserva natural, monumento natural, paisaje protegido, zona de interés regional y corredor ecológico y de biodiversidad), planes de gestión para la conservación y ocio (parque periurbano de conservación y ocio), normas de conservación (lugares de interés científico y árbol singular) y planes especiales (corredor ecocultural). En la actualidad, los dos parques naturales (Cornalvo y Tajo Internacional) y la reserva natural de la Garganta de los Infiernos disponen de un plan de ordenación de los recursos naturales aprobado. Por su parte, los planes rectores de uso y gestión aprobados afectan a los siguientes espacios: Parque Natural de Cornalvo, Parque Natural del Tajo Internacional, Reserva Natural de la Garganta de los Infiernos, Zona de Interés Regional Llanos de Cáceres y Sierra de Fuentes, Zona de Interés Regional Embalse de Orellana-Sierra de Pela y Zona de Interés Regional Sierra de San Pedro. Estos instrumentos resultan fundamentales a la hora de marcar los usos compatibles e incompatibles de los diferentes espacios, en un escenario en el que los espacios naturales protegidos se han convertido en áreas donde se llevan a cabo numerosas actividades recreativas.

En el caso de la Red Natura, la reciente aprobación del Decreto 110/2015 incluye como instrumentos de gestión de los espacios, el Plan Director de la Red Natura 2000, los Planes de Gestión y otros instrumentos de gestión. 
Figura 1. Instrumentos de ordenación y planificación.

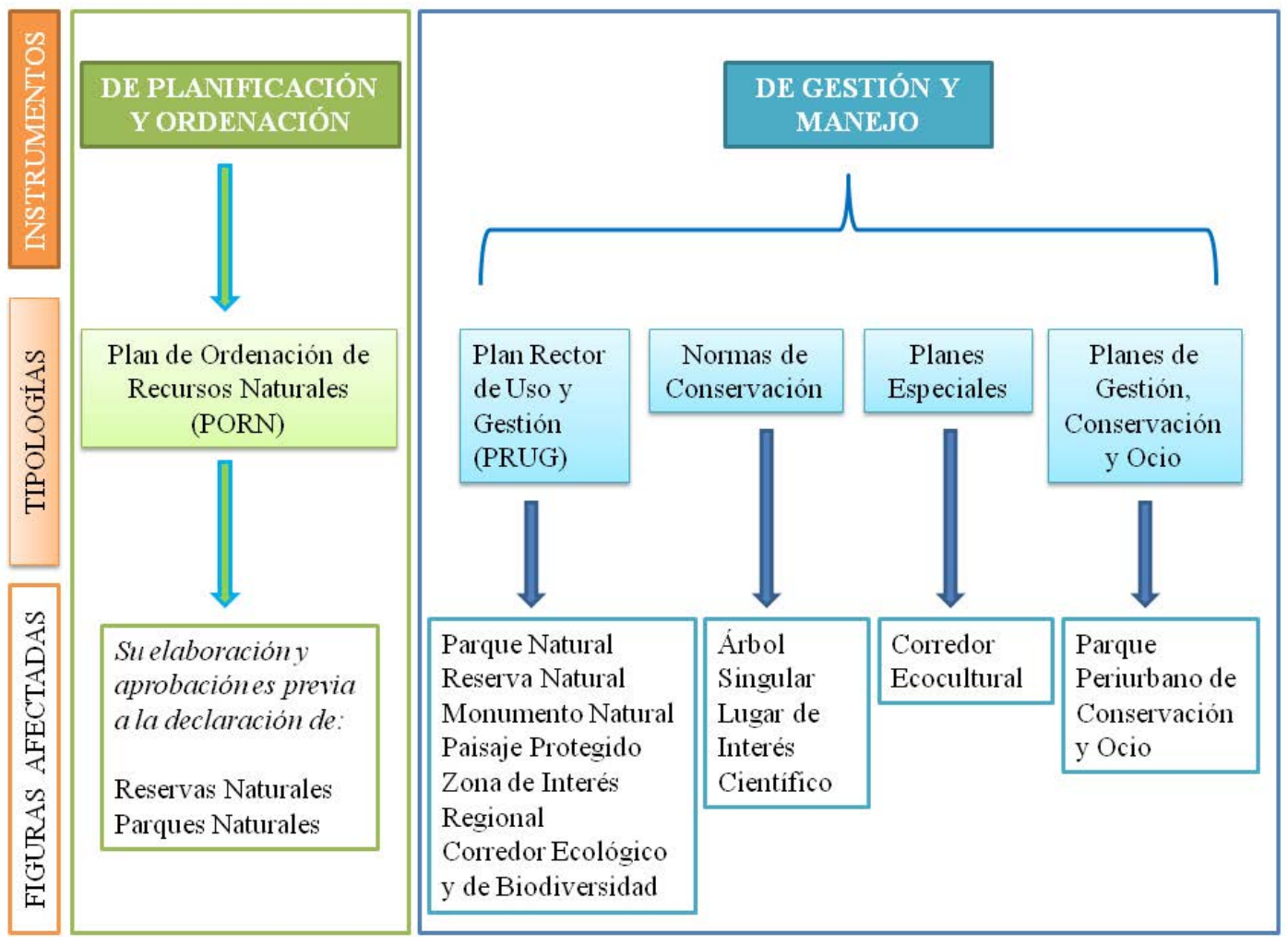

Elaboración propia.

\section{LA ACTIVIDAD CINEGÉTICA EN EL CONTEXTO ACTUAL}

La caza se ha percibido de formas muy diferentes a lo largo de la historia. Por ello, la visión que el hombre pueda tener de la caza en el siglo XXI es, a todas luces, muy distinta de la que poseería alguien que vivió en otros periodos históricos, aun a pesar de que el elemento consustancial que la actividad cinegética encierra en su concepción, el apresamiento del animal, siempre haya permanecido vigente desde sus orígenes. Así lo recogió Martínez de Espinar (1644) en el siglo XVII cuando definió a la caza como "... vna acción de bufcar, feguir, y perfeguir a las fieras o a las aves para rendirlas y fujetarlas el hombre a su dominio"; y así lo expresaron, por una parte, las viejas normas de caza de los años 1879, 1902 y 1970 y, por otra, las leyes cinegéticas más recientes sancionadas por las comunidades autónomas en el período democrático, tras la cesión de competencias por parte del estado central: “...la ejercida mediante el uso de armas, animales, artes y otros medios para buscar, atraer, perseguir, capturar o acosar a los animales definidos por esta ley como piezas de caza, con el fin de darles muerte, atraparlos, apropiarse de ellos o de facilitar su captura por un tercero, así como la ejecución de los actos preparatorios que resulten necesarios a tal fin" (Ley 14/2010, de 9 de diciembre, de Caza de Extremadura).

En suma, si estas percepciones no han sido idénticas en el devenir de los tiempos se debe a que la caza ha estado inmersa, a escala global, en cambios constantes que se pueden sintetizar en los siguientes aspectos:

- La profunda evolución que han sufrido las técnicas empleadas para cazar. Se ha pasado de la incertidumbre que durante siglos caracterizó a las herramientas utilizadas para ejercitar las artes venatorias, a la certidumbre, garantía de éxito, que proporcionan los ingenios actuales, con la complicidad de los sistemas de manejo y gestión. En síntesis, armas más perfectas, técnicas más depuradas y, en ocasiones, planes de manejo de las especies venatorias que proporcionan mayores ventajas al cazador, como pueden ser los cercados. A ello habría que sumar los avances que ha experimentado la sociedad en términos de motorización, mejora de la accesibilidad, disposición de tiempo libre y aumento de rentas. Progresos que se vieron acompañados, desde principios del siglo XX, de un aumento del 
número de cazadores. Por último, mientras el proceso evolutivo se manifestaba de la forma en que se ha expuesto anteriormente, el sector de la caza se vio afectado por el proceso de mundialización que envolvió a numerosas actividades, fase en la que actualmente se encuentra.

- Los cambios experimentados en las finalidades perseguidas, cuya deriva ha seguido un itinerario que ha ido de la caza útil a la recreativa y de la búsqueda de objetivos tangibles a otros en que se persigue a la pieza por recreación o placer (Leader-Williams, 2009). La caza fue, primero, una actividad con la que el hombre contribuía a su sustento, pero ya en la Edad Media las finalidades que perseguían los cazadores tuvieron un carácter dual. Por una parte, los fines utilitarios (proporcionar alimentos, control de las poblaciones de algunos animales o preparación para la guerra) y, por otra, los que perseguían el entretenimiento. Habría que esperar al siglo XX para que las finalidades pretendidas con la caza fueran mayoritariamente recreativas, asumiendo un preeminente rol de actividad que llenaba parte del tiempo libre destinado al ocio. Este cambio se manifestó, de un modo más prematuro y evidente, en los países desarrollados. De hecho, tan solo en algunas zonas y países en desarrollo sobrevive con carácter residual, como en el Oeste de África, un tipo de caza que da preferencia a la obtención de carne (Caspary, 2001). Obviamos, por supuesto, las prácticas cinegéticas al margen de la Ley, donde los furtivos pueden cazar por motivaciones muy diversas, principalmente de carácter económico.

- Las reglas de juego establecidas por un marco legal complejo, donde la consabida soberanía nacional, en materia legislativa, debe contener aspectos que emanan de la firma de convenios internacionales tras el firme compromiso adquirido por los diferentes estados para proteger a la fauna (Convenio relativo a la Conservación de la Vida Silvestre y del Medio Natural en Europa -Berna-, Convention on International Trade in Endangered Species of Wild Flora and Fauna -CITES-, Convention on the Conservation of Migratory Species of Wild Animals -CMS-, Convention on Wetlands -Ramsar-). En este sentido, la caza, como actividad consuntiva de recursos silvestres renovables, se legisla en el tiempo presente en clave de ejercicio que se puede practicar en términos de sostenibilidad y de contribución a la conservación de la naturaleza. Esta consideración se alimenta de un nuevo rumbo ideológico que toma como punto de partida lo indicado en el convenio sobre la diversidad biológica, nacido de la Cumbre de la Tierra celebrada en Río de Janeiro en el año 1992, cuyos objetivos reflejan una ética que entiende como posible el uso sostenible de los recursos naturales (Convention on Biological Diversity, 2003). Años más tarde, los principios y directrices de Addis Abbeba para el uso sostenible señalaron que el uso de los recursos silvestres, si se hacía bajo las condiciones de un adecuado manejo, se convertía en una herramienta válida para el mantenimiento de la diversidad biológica (Convention on Biological Diversity, 2004). Por este motivo se ha llegado a acuñar el concepto de caza sostenible, entendida como el uso de las especies de caza y sus hábitats de una forma y a un ritmo que no lleve a la disminución a largo plazo de la diversidad biológica y satisfaga las necesidades y aspiraciones de las generaciones presentes y futuras (Council of Europe, 2007). En este caso, la práctica cinegética se transforma en una herramienta útil para la conservación.

En consecuencia, este proceso ha transformado a la actividad cinegética en un ejercicio que, por una parte, se practica de acuerdo con los principios de la gestión sostenible y, por otra, en un fenómeno que ha alcanzado sobresalientes repercusiones sociales, económicas y espaciales a diferentes escalas. Bajo este contexto, la actividad cinegética genera indudables beneficios directos e indirectos en distintos planos, siendo destacables los que se insertan en los órdenes económico y ambiental. Los beneficios ambientales, principalmente los asociados a la conservación de la naturaleza, forman parte del discurso que esgrimen los defensores de la caza para responder a grupos contrarios a la caza que cuestionan la legitimidad de la caza recreativa por motivaciones de índole ética (sufrimiento de los animales) y conservación (potencial amenaza para las especies). Asimismo, de la caza se desprenden otros beneficios ecológicos. En este sentido, la conservación de no pocos espacios naturales de Europa ha alcanzado altas cotas gracias a los intereses ligados al aprovechamiento cinegético (Comisión Europea, 2008). En España, de forma clara, la caza fue la razón por la que se llegaron a proteger/acotar espacios como Gredos, Cabañeros, Picos de Europa etc. Incluso se puede señalar que la protección de los bosques, en el periodo previo a la aparición de los primeros espacios naturales protegidos, estuvo en muchos casos vinculada con los intereses cinegéticos o madereros (European Environment Agency, 2012). En cuanto a los beneficios económicos son palpables en todos aquellos sectores que se relacionan, directa o indirectamente, con el cazador en su faceta de consumidor (armas, cartuchos, ropa, hostelería, óptica, publicaciones, complementos), así como entre 
los propietarios y gestores de los terrenos de caza, favoreciendo la creación de empleo (Bernabéu Cañete, 2002). La caza tiene especial interés económico para determinadas explotaciones agrícolas y ganaderas que ingresan por este concepto rentas de mayor o menor cuantía, contribuyendo a su mantenimiento. En otras partes, como África, la gestión de los recursos cinegéticos por parte de las comunidades locales, ha proporcionado estímulos positivos para el uso sostenible de los recursos, como consecuencia de la recepción de beneficios económicos (Baker, 1997). Beneficios que les han proporcionado el acceso a bienes y servicios que han mejorado su nivel de bienestar (Jones, 2009).

En el caso concreto de España, la actividad cinegética se ha visto afectada por los procesos descritos anteriormente. Por ello, durante el siglo XX la caza en España se ha convertido en una actividad recreativa que se ha caracterizado por lo siguiente:

- Crecimiento exponencial del número de cazadores que se intensificó en la década de los sesenta y setenta, razón por la que este proceso ha sido denominado por algunos autores como un boom cinegético (López Ontiveros, 1991a). Este crecimiento se truncó a finales de la pasada centuria, tal y como puede constatarse estadísticamente en diferentes escenarios geográficos (Rengifo Gallego, 2012).

- Mercantilización de la actividad cinegética como consecuencia del aumento de la demanda, del incremento de la superficie acotada, de la aplicación de métodos de gestión y la profesionalización. Asimismo, la caza empieza a considerarse como un recurso turístico con capacidad para a atraer a visitantes (Borrell, 1964; Muñoz-Goyanes, 1964; Rengifo Gallego, 2008, 2009) y, por ello, surgirá en 1977 la primera empresa española turístico cinegética para prestar servicios a cazadores extranjeros (Medem SanJuan, 2002).

- Asunción en masa de las competencias en materia de caza por parte de las Comunidades Autónomas durante la década de los 80 , circunstancia que contribuyó a la aparición de numerosas normas específicas de caza que hicieron más complejo el corpus normativo. Igualmente, este importante cambio supuso una pérdida de la perspectiva global de la actividad cinegética a escala nacional. Por este motivo, se puede señalar que ha existido en las últimas décadas un déficit de trabajos que aborden, bajo una visión general, la actividad cinegética en España. Muy lejanos quedan ya los trabajos realizados por geógrafos en la década de los noventa, en los que prevaleció el estudio de la caza bajo esa perspectiva global y con diferentes enfoques (López Ontiveros 1991a, 1991b, 1994; López Ontiveros y García Verdugo, 1991; Mulero Mendigorri 1991; López Ontiveros y Mulero Mendigorri, 1998). Más cercanos se sitúan algunos estudios llevados a cabo por otros geógrafos en los que se abordan a escala estatal algunos aspectos específicos relacionados con la caza, como el turismo cinegético (Rengifo Gallego, 2009) o la perspectiva territorial referida al periodo 1970-1987 (Martínez Garrido, 2009). Tampoco se pueden ignorar los recientes trabajos de investigadores de otras disciplinas que han analizado la evolución de algunas variables esenciales del sector. Se trata de los trabajos de Herruzo y Martínez-Jáuregui (2013), y de Martínez-Jáuregui, Arenas y Herruzo (2011), referidos a la evolución del número de licencias, cotos y especies en las dos últimas décadas.

\subsection{Referencias a la actividad cinegética en Extremadura}

La fortaleza de la práctica cinegética de Extremadura, en el contexto actual, se hace palpable tras la evaluación de las principales variables en las que se sustenta el ejercicio venatorio. Una radiografía de las principales variables que son imprescindibles para el desarrollo de la caza a escala regional, pone de manifiesto la siguiente realidad:

- Terrenos cinegéticos. La mayor parte de la superficie de Extremadura tiene la consideración de terreno cinegético, viéndose afectada por algunas de las tipologías contempladas en la legislación vigente: terrenos cinegéticos bajo gestión pública y cotos de caza, principalmente (tabla 3). El aspecto más destacado es el alto porcentaje de superficie acotada, superior al 80\%, y la convivencia de dos tipologías de terrenos cinegéticos, de acuerdo con su funcionalidad social y económica: terrenos cinegéticos cuya finalidad es eminentemente social y deportivo (cotos sociales) y terrenos en los que el propósito es el aprovechamiento de los recursos cinegéticos con una finalidad comercial o de carácter privado (cotos privados). Asimismo, la gestión de los terrenos cinegéticos corresponde, casi en su totalidad, al sector privado. 
Tabla 3. Terrenos de caza en Extremadura. Temporada 2014/15

\begin{tabular}{|c|c|c|c|c|}
\hline & Número & $\%$ & Hectáreas & $\%$ \\
\hline Sociales & 624 & $15,61 \%$ & 1.693 .932 & $47,23 \%$ \\
\hline Privados de caza menor extensivos & 1.326 & $33,17 \%$ & 709.436 & $19,78 \%$ \\
\hline Privados de caza menor más jabalí & 521 & $13,03 \%$ & 267.463 & $7,46 \%$ \\
\hline Privados de caza menor intensivos & 110 & $2,75 \%$ & 71.987 & $2,01 \%$ \\
\hline Privados de caza mayor abiertos & 647 & $16,19 \%$ & 541.846 & $15,11 \%$ \\
\hline Privados de caza mayor cerrados & 165 & $4,13 \%$ & 170.508 & $4,75 \%$ \\
\hline Refugios para la caza & 7 & $0,18 \%$ & 895 & $0,02 \%$ \\
\hline ZCL cerradas & 591 & $14,79 \%$ & 73.685 & $2,05 \%$ \\
\hline TT.CC. Gestión pública & 6 & $0,15 \%$ & 57.032 & $1,59 \%$ \\
\hline Total & 3.997 & & 3.586 .784 & \\
\hline
\end{tabular}

Fuente: Junta de Extremadura. Elaboración propia.

- Especies de caza. La diversidad de especies cinegéticas se ve reflejada en las órdenes que aprueba la Consejería competente en la materia. Del análisis realizado por Rengifo Gallego (2012), a partir de las órdenes que regulan la caza, se desprende que el número de especies de caza menor se ha mantenido en los últimos años por encima de la veintena. Estas especies las forman, en su mayor parte, aves (migratorias y sedentarias) y tres mamíferos (conejo, liebre y zorro). El número de capturas de estas especies alcanzan importantes dígitos cada temporada de caza, especialmente entre las especies de perdiz, conejo, liebre, codorniz y paloma torcaz. Las especies de caza mayor, por su parte, son siete (jabalí, ciervo, gamo, cabra montés, corzo, muflón y arruí). En cuanto a las capturas de las especies de caza mayor destacan, sobre las demás, las de jabalíes y ciervos (tabla 4), presentando unas cifras que, en términos comparativos con el resto de comunidades españolas, son de las más altas (Rengifo Gallego, 2009). La tendencia de las capturas de estas especies en los últimos años muestra una trayectoria alcista que, según parece, se va a mantener a corto plazo, en detrimento de algunas especies de caza menor.

Tabla 4. Capturas de la temporada 2014/15

\begin{tabular}{|l|r|r|r|}
\hline \multicolumn{1}{|c|}{ Especie } & \multicolumn{1}{c|}{ Badajoz } & \multicolumn{1}{c|}{ Cáceres } & Extremadura \\
\hline Ciervo & 9.037 & 21.126 & 30.163 \\
\hline Jabalí & 7.446 & 11.946 & 19.392 \\
\hline Corzo & 63 & 424 & 487 \\
\hline Cabra Montés & -- & 379 & 379 \\
\hline Gamo & 1092 & 1280 & 2372 \\
\hline Muflón & 415 & 849 & 1264 \\
\hline Conejo & 42.356 & 22.565 & 64.921 \\
\hline Liebre & 79.381 & 21.368 & 101.199 \\
\hline Perdiz & 141.925 & 90.292 & 232.217 \\
\hline Paloma Torcaz & 21.126 & 21.368 & 42.494 \\
\hline Zorzal & 323.487 & 204.465 & 527.952 \\
\hline Codorniz & 33.831 & 8.737 & 42.568 \\
\hline Acuáticas & 16.249 & 8.650 & 24.899 \\
\hline Zorro & 19.931 & 15.578 & 35.509 \\
\hline
\end{tabular}

Fuente: Junta de Extremadura. Elaboración propia.

- Número de cazadores. El colectivo de cazadores alcanza valores absolutos elevados (78.882 licencias expedidas en 2009), muchos de los cuales pertenecen a alguna de las sociedades de cazadores existentes a escala local. De hecho, el número de licencias de caza, en relación con la población absoluta, presenta valores elevados en comparación con la mayor parte de los países europeos y regiones espa- 
ñolas. Sin embargo, se aprecia que la distribución de los poseedores de licencias por grupos de edad presenta un prominente grado de envejecimiento, por lo que este dato no deja de ser inquietante ante la necesidad de garantizar el relevo generacional. Asimismo, los cazadores se decantan por la opción de cazar, mayoritariamente, con armas de fuego, tal y como demuestra el dato de que la las licencias de clase A (las que habilitan para cazar con armas de fuego) sean, con diferencia, las más numerosas. Por último, del análisis del lugar de residencia de los poseedores de licencia se desprende que alrededor del $80,0 \%$ son residentes extremeños y el resto tienen fijada su residencia en otras comunidades autónomas y el extranjero.

- Economía de la caza. Hay un evidente déficit en cuanto a la elaboración de trabajos que aporten conocimiento riguroso sobre la economía que se genera alrededor de la actividad cinegética en Extremadura. Aunque la administración regional maneja cifras que airea en los medios de comunicación (300-400 millones de euros), no son más que estimaciones que recientemente han sido refrendadas por el Plan General de Caza de Extremadura, documento que recoge una estimación de 332,7 millones de euros (Junta de Extremadura, 2015). Lo que no suscita duda alguna es la trascendencia que la actividad cinegética tiene para las economías rurales, fruto del papel que asume el cazador en calidad de consumidor de diferentes tipos de servicios, como los de la rama de hostelería, y en calidad de adquiriente de una amplia relación de equipamientos que hacen factible la actividad cinegética: armas, cartuchería, vehículos especializados, taxidermia, perros y diferentes tipologías de complementos.

En síntesis, a tenor de los datos expuestos se colige que la caza en Extremadura está ampliamente extendida y que es una realidad que tiene importantes repercusiones territoriales, sociales y económicas.

\section{CAZA Y ESPACIOS PROTEGIDOS EN EXTREMADURA}

Los datos expuestos sobre la situación actual de la actividad cinegética en Extremadura, no hacen sino confirmar la pervivencia de esta actividad a lo largo de la historia, como resultado de la existencia de tres factores primordiales en la región: fuerte arraigo social, mantenimiento de unas favorables condiciones ecológicas y estructura de la propiedad. Con estos antecedentes, la actividad cinegética en Extremadura se ha visto afectada, desde el año 1979, fecha de la declaración del primer espacio protegido a escala regional, por un proceso que ha culminado, en poco más de 25 años, con la protección de casi un tercio de la superficie regional. Como consecuencia de ello, ha surgido un nuevo escenario en el que los intereses cinegéticos, que afectan a una extensión superficial muy elevada, se han visto obligados a compartir el mismo territorio con otros de características muy diferentes (conservacionistas, turísticos etc.), máxime cuando se da la circunstancia de que muchos espacios naturales protegidos se distribuyen por territorios en los que los recursos cinegéticos han sido abundantes y, por ende, gozan de una larga tradición venatoria. Ante esta situación, han aparecido nuevos enfoques que tratan de limar los conflictos derivados de la gestión de la caza con la conservación. Uno de estos enfoques es el de la custodia del territorio: "una nueva metodología de manejo de los valores naturales, culturales y paisajísticos que parte de un enfoque integrador y que cuenta con la participación activa de los agentes sociales, tanto públicos como privados, del propio territorio" (Martínez, Sánchez y Torija, 2011, p. 249).

En Extremadura la superposición entre zonas protegidas y zonas de caza emblemáticas ofrece ejemplos muy evidentes que aparecen señalados en la Figura 2: Sierra de San Pedro (coincidente con una Zona de Interés Regional y espacios de Natura 2000), Monfragüe (Parque Nacional, Natura 2000, Reserva de la Biosfera), Sierra de Hornachos (Natura 2000), Riberos del Tajo Internacional (Parque Natural, Natura 2000), Villuercas-Ibores-Jara (Natura 2000), La Siberia (Natura 2000). En todos ellos, la superficie acotada alcanza valores significativos, en correlación con el dato de que en Extremadura están acotadas 3,4 millones de hectáreas que vienen a representar algo más del 82\% de la superficie regional (Junta de Extremadura, 2014). Así lo confirma el Plan General de Caza (Junta de Extremadura, 2015), donde en la comarcalización propuesta se aprecia la ingente cantidad de cotos que hay en espacios como Sierra de San Pedro-Tajo Internacional (293 cotos y 304.073 hectáreas) con la consiguiente dependencia económica que tienen de la caza numerosas explotaciones en las que escasean otros tipos de aprovechamientos. Estas circunstancias no son exclusivas de Extremadura ya que la superposición de figuras de protección con zonas de caza tradicionales es una constante que se repite en otras zonas de España, tal y como queda patente en algunos trabajos recientes referidos a Andalucía (Mulero Mendigorri, 2013). 
Figura 2. Distribución de la superficie protegida de Extremadura, zonas de caza tradicionales y terrenos cinegéticos gestionados por la administración

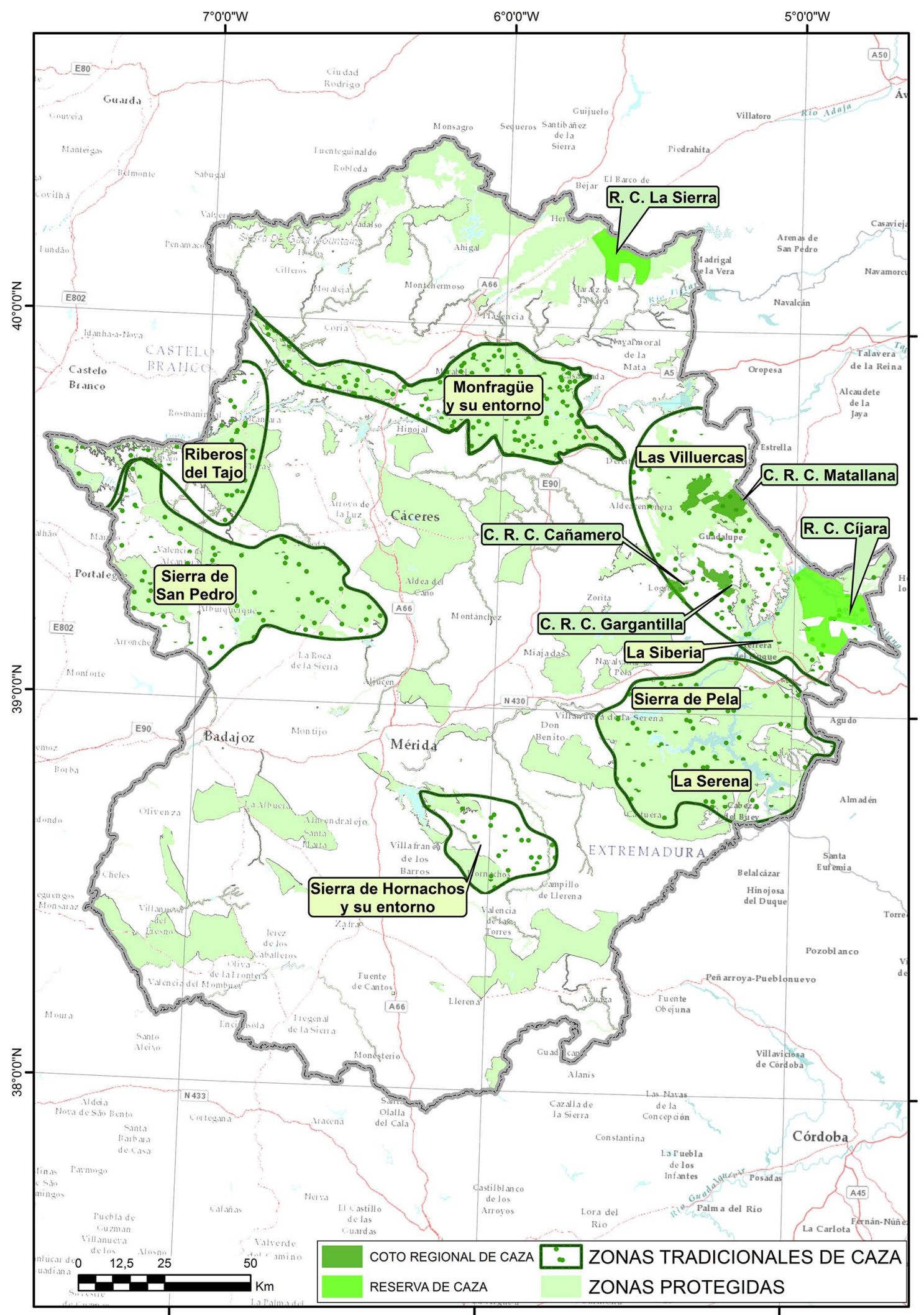

Fuente: ESRI, DeLorme, USGS, NPS. Elaboración propia. 
Igualmente, se da la circunstancia de que los actuales terrenos de caza gestionados por la administración regional se localizan en áreas de gran interés ecológico que, en la mayor parte de los casos, coinciden con terrenos protegidos. Esta coincidencia puede advertirse, de nuevo, en la Figura 2. Los terrenos cinegéticos a los que se alude son los siguientes:

- Reservas de Caza. En Extremadura se encuentran dos importantes reservas de caza: Cíjara y La Sierra. La Reserva de Caza del Cíjara (22.187 has.), situada en el Noreste de la provincia de Badajoz, fue creada por Ley 37/1966, de 31 de mayo, sobre creación de Reservas Nacionales de Caza. Dicha norma se situó como un claro precedente legislativo en términos de caza-conservación, en el que se expresaba de forma explícita que la creación de las Reservas de Caza permitiría compaginar la utilización racional de los recursos, al tiempo que contribuiría a "promover la máxima satisfacción social, económica y recreativa que la Naturaleza y los seres que la pueblan puedan proporcionar a una comunidad". Aquella Ley creó 20 Reservas Nacionales de Caza distribuidas por 14 provincias españolas, siendo ampliada la red en el año 1973 mediante nueva Ley 2/1973, de 17 de marzo, de creación de 13 reservas nacionales de caza. La Reserva de Caza La Sierra (13.098 has.), por su parte, fue creada en la etapa en la que las comunidades autónomas habían recibido ya las competencias en materia de caza, al amparo de lo dispuesto en la Ley 8/1990 de Caza de Extremadura. Estas nuevas Reservas de Caza se concebían, de acuerdo con lo dictado por la Ley, como "núcleos de excepcionales posibilidades cinegéticas" en los que la finalidad era "promover, conservar, fomentar y proteger determinadas especies cinegéticas y sus hábitats, subordinando su posible aprovechamiento a dicha finalidad y, en su caso, a la crianza para repoblar de forma natural otros terrenos cinegéticos" (Artículo 15).

- Cotos Regionales de Caza. Tienen como finalidad facilitar el ejercicio de la caza en igualdad de oportunidades y con especial atención a los cazadores extremeños. Los exponentes de esta figura son el Coto Regional de Gargantilla (4.737 has.), Coto Regional de Matallana (10.303 has.) y Coto Regional de Cañamero (2.064 has.). Todos ellos se distribuyen por la comarca de las Villuercas, situada al este de la provincia de Cáceres.

Mientras tanto, entre las actividades que han irrumpido con fuerza en los ENP, durante los últimos años, se encuadran las turísticas. Los ENP vienen demostrando que funcionan como centros receptores de visitantes, en virtud de su capacidad para atraer a personas que se desplazan atraídas por los recursos existentes en sus territorios. Por estos motivos, las cifras de visitantes que vienen registrando algunos de estos ENP han crecido de forma acelerada. Ejemplo de ello son los Parques Nacionales, tipología de espacio que, de acuerdo con los datos disponibles para el periodo 1984-2005, han experimentado un incremento considerable de visitas, cifrada en un 348\% para la serie completa reseñada (Muñoz Santos y Benayas del Álamo, 2012). En Extremadura, el Parque Nacional de Monfragüe recibe, con carácter anual, un número de visitantes muy importante que ha oscilado entre los 272.683 del año 2012 y los 351.885 del año 2007.

Por su parte, los visitantes que reciben los diferentes equipamientos ambientales de la región, vinculados en la mayor parte de los casos a los ENP y espacios de la Red Natura 2000, reflejan lo mismo. Durante el año 2012 los centros de interpretación de la naturaleza recibieron la visita de 167.673 personas (Junta de Extremadura, 2014). Este crecimiento del número de visitantes de los ENP se ha producido en un contexto de crecimiento del turismo en las áreas rurales de Extremadura, proceso que ha sido bien acogido por los agentes turísticos públicos y privados ante las posibilidades que ofrece como actividad cualificada para impulsar el desarrollo y mitigar algunos de los problemas de los que adolecen estos espacios.

A la luz de esta incontestable realidad, se hace necesario estudiar si realmente se están produciendo conflictos de intereses entre el aprovechamiento cinegético tradicional y las políticas de protección, más allá del debate que tratan de impulsar los grupos ecologistas contrarios a la caza. No se puede ignorar que la caza y los espacios naturales han chocado alrededor de dos cuestiones: el carácter consuntivo de la actividad cinegética y la aplicación de algunas medidas de gestión que producen impactos sobre el territorio.

En este sentido, hay que afirmar que en Extremadura no se produjeron colisiones de intereses entre la caza y la conservación hasta el año 1991, fecha en la que entró en vigor la polémica Ley de Caza 8/1990 en la que se prohibió de forma explícita la actividad cinegética en los Parques Naturales, de acuerdo con lo señalado en su artículo 13: 
En los Parques Naturales está prohibido, con carácter permanente, el ejercicio de la caza, salvo cuando por razones de orden biológico, técnico o científico, debidamente justificadas, la Agencia conceda la oportuna autorización, fijando las condiciones aplicables a cada caso.

Este nuevo rumbo ideológico, de indudable carácter restrictivo e intervencionista, fue altamente contestado entre los propietarios de terrenos de caza afectados, así como entre los colectivos de cazadores, generando un incontable número de reacciones en contra de la medida. Aquella disposición, con el discurrir de los años, arrastró una serie de consecuencias que tuvo efectos nocivos para la propia conservación del Parque. Uno de ellos fue la superpoblación de ungulados silvestres en Monfragüe -jabalíes y ciervos- que impactó negativamente en dos sentidos: daños a la flora del parque y propagación de enfermedades que acabaron afectando a la cabaña ganadera de la zona.

Ocho años después de la aprobación de la primera Ley de Caza, la prohibición de cazar en los Parques Naturales fue refrendada, en la misma dirección, por la Ley 8/1998 de Conservación de la Naturaleza y de los Espacios Naturales Protegidos de Extremadura. No obstante, aquella Ley marcó un nuevo punto de partida en relación con los usos y aprovechamientos factibles de ser llevados a cabo en los ENP. La razón no fue otra que la inclusión, en el marco normativo de la Comunidad Autónoma, de lo que se denominan instrumentos de ordenación y planificación en los que con el paso del tiempo se depositó la capacidad de autorizar y regular los usos y aprovechamientos compatibles con la conservación.

Sin embargo, habría que esperar al año 2006 para que se diera un paso crucial en la profundización de esta iniciativa, tras la modificación de la Ley 8/1998. La nueva norma (Ley 9/2006 de 23 de diciembre) recogería un artículo donde la prohibición de cazar en los Parques Naturales adquirió un nuevo e importante matiz al quedar redactado de la siguiente forma:

En los Parques Naturales no se permitirá el ejercicio de la caza, salvo que expresamente se autorice y regule en sus instrumentos de planificación, manejo y gestión o cuando, por razones de orden biológico, técnico o científico, el órgano competente en materia de medio ambiente conceda la oportuna autorización.

Con la redacción de esta nueva Ley en el horizonte, los ENP se veían abocados a la redacción de unos Planes en los que la actividad cinegética debía ser abordada necesariamente, por ser una cuestión ineludible. Sin duda, con la experiencia acumulada, tras la inicial prohibición de cazar en los Parques Naturales desde el año 1991, se recondujo la situación hacia posturas menos rígidas, tal y como recogieron los diferentes tipos de instrumentos redactados:

- Instrumentos de planificación y ordenación: Planes de Ordenación de Recursos Naturales -PORN(su aprobación es obligatoria y previa para los Parques Naturales y Reservas Naturales). En estos planes recaen, entre otras atribuciones, las limitaciones generales y específicas que respecto de los usos y actividades hayan de establecerse en función de la conservación de los espacios y especies que proteger, con especificación de las distintas zonas en su caso (Zonas de uso restringido, limitado, compatible y general). En la actualidad están aprobados el 100\% de los PORN exigidos por Ley (Junta de Extremadura, 2014): Parque Natural del Tajo Internacional, Parque Natural de Cornalvo y Reserva Natural Garganta de los Infiernos. Del análisis de sus contenidos se desprende que si bien los primeros PORN publicados prohibían taxativamente las actividades cinegéticas, como ocurrió con el PORN del Parque Natural de Cornalvo del año 2005 (Artículo 13 referido a limitaciones específicas), con posterioridad se ha optado por permitir la caza ordenada y sostenible, tal y como recoge, por ejemplo, el recientemente aprobado PORN del Parque Natural del Tajo Internacional. No obstante, de su lectura se desprende que se establecen diferentes limitaciones y se lleva a cabo un mayor control sobre algunas de las acciones de caza, circunstancias que convierten a estos espacios, sin duda, en territorios que reciben un trato diferencial. Por último, la lectura del PORN de la Reserva Natural de la Garganta de los Infiernos deja claro que se permiten los aprovechamientos cinegéticos conforme a lo dispuesto por la normativa específica y los Planes de ordenación y aprovechamiento cinegético. Se reconoce la existencia de una subzona cinegética dentro de la zona de uso limitado, coincidente con los terrenos de la Reserva de Caza la Sierra, si bien se permite la caza en todas las subzonas que componen la zona de uso limitado.

- Instrumentos de gestión y manejo: Planes Rectores de Uso y Gestión -PRUG- (obligatorios para Parques Naturales, Reservas Naturales, Monumentos Naturales, Paisajes Protegidos, Zonas de Interés Regional y Corredores Ecológicos y de Biodiversidad). Hasta el momento, han sido aprobados siete 
PRUG que afectan a los Parques Naturales de Cornalvo y Tajo Internacional, Reserva Natural de la Garganta de los Infiernos y Zonas de Interés Regional de Los Llanos de Cáceres, Sierra de San Pedro y Embalse de Orellana-Sierra de Pela. Por tanto, hay un evidente déficit en la aprobación de estos instrumentos, ya que todavía son numerosos los espacios que no cuentan con un PRUG, aun a pesar de que la Ley estableció unos plazos que no siempre se han cumplido. Para conocer cómo aparece reflejada la actividad cinegética en los documentos, se han analizado los correspondientes a las tres Zonas de Interés Regional que disponen de este instrumento aprobado, por corresponderse con áreas de gran interés cinegético y tener una considerable superficie: Sierra de San Pedro, Llanos de Cáceres-Sierra de Fuentes y Embalse de Orellana-Sierra de Pela. En los tres PRUG la caza se contempla en clave de actividad que debe ser compatible con el mantenimiento de los recursos naturales y con otros usos. Asimismo, en la Sierra de San Pedro, territorio de especial vocación cinegética, se apuesta por el fomento de la caza como actividad necesaria y modo de control de las poblaciones cinegéticas y medio para obtener recursos económicos. En cuanto a las medidas relacionadas con la gestión se plantean en base a objetivos que persigan, básicamente, lo siguiente:

- Mejora y mantenimiento de los ecosistemas, con especial énfasis en el respeto a los ciclos de las diferentes especies, sean cinegéticas o no.

- Adecuación de los Planes Técnicos de caza.

- Realización sistemática de censos de animales cinegéticos.

Al margen de los instrumentos de planificación que figuran en las normas puramente ambientales, hay que incidir que la normativa específica de caza también dispone de unos planes que tienen por finalidad hacer de la actividad cinegética, una práctica sostenible. A escala de los cotos de caza el instrumento de gestión es el plan técnico, cuya finalidad es garantizar el aprovechamiento ordenado y sostenible de los recursos de caza, sin menoscabar la conservación de la fauna silvestre y sus ecosistemas.

\subsection{La especial situación de Monfragüe}

El caso de Monfragüe, tras ser declarado durante el año 2007 Parque Nacional, debe ser objeto de un tratamiento diferente, al verse afectado por la Ley de Parques Nacionales (Ley 30/2014 de Parques Nacionales) en la que se expresa la incompatibilidad, con los fines propios de la conservación, "de la caza deportiva y comercial". Dicha medida, que cuenta con el beneplácito de las asociaciones ecologistas, no es compartida por los colectivos de cazadores. Asimismo, hay que señalar que esta controvertida medida cuenta en Monfragüe con dos razones que dificultan su aplicación:

- Alto porcentaje de superficie en manos de propietarios privados. Aunque en la mayor parte de los Parques Nacionales la tónica general es la predominancia de suelo público frente al privado, en Monfragüe la situación es diferente al alcanzar la superficie privada el 54,62\%. El resto de la superficie pertenece a diferentes instituciones públicas: estatal $(30,8 \%)$, autonómica $(5,56 \%)$ y municipal $(9,73 \%)$ (OAPN, 2013).

- Abundancia de ungulados silvestres. La abundancia de ciervos y jabalíes es una realidad que obliga a controlar sus poblaciones para evitar daños a la flora, propagación de enfermedades y accidentes. El PORN de Monfragüe, del año 2005, reconocía dicha circunstancia y señalaba que para evitar estos problemas era eficaz la caza selectiva "como mejor instrumento para evitar el incremento desproporcionado de las poblaciones de ciervo y jabalí, con el fin de evitar la erosión del suelo y la destrucción irreversible de la vegetación autóctona, además de considerarse un instrumento de desarrollo socioeconómico para el conjunto de las poblaciones del entorno del área protegida". Como la expresión caza selectiva entraña diferentes interpretaciones el PRUG del año 2014 clarificó el tipo de acciones que podrían llevarse a cabo en el caso de que las densidades de ungulados pudieran afectar al buen estado de conservación de las formaciones vegetales. En este sentido, el PRUG establece la necesidad de desarrollar un Programa de Acción Selectiva conforme a las directrices básicas establecidas en un Plan de Acción Selectiva, que se ha de basar en estudios científicos o técnicos, y datos actualizados relativos a las densidades de ungulados en el Parque.

\section{CONCLUSIONES}

La historia de la protección de los espacios naturales protegidos cuenta con una trayectoria que, aunque no es dilatada en el tiempo, ha sido intensa, especialmente desde la fecha en la que se celebró la 
Cumbre de Río. La realidad actual nos señala que en este periodo la superficie terrestre protegida, a escala mundial, ha alcanzado la cifra del 14,6\%, a la que habría que añadir un 9,7\% de aguas costeras. Por su parte, en Europa, el porcentaje de superficie protegida consiguió cifras relevantes en un periodo corto de tiempo, aunque con notables diferencias entre países, primordialmente tras el desarrollo de la Red Natura 2000 y las figuras que se incluyen en ella.

Extremadura comenzó su andadura proteccionista en el año 1979, tras la declaración de Monfragüe como Parque Natural. No obstante, habría que esperar a la década de los noventa de la pasada centuria y primeros años del actual siglo, para que la superficie protegida creciera de forma significativa, hasta superar el 30\% del territorio regional, distribuida entre una extensa relación de figuras que contempla el marco normativo. Como resultado de ello, se han generado nuevas condiciones en lo que a usos y aprovechamientos se refiere en los espacios que han sido protegidos, siendo la actividad cinegética uno de los aprovechamientos tradicionales afectados. En este sentido, hay que señalar que la caza en Extremadura es una actividad que goza de una larga tradición, debido a la pervivencia de una serie de condiciones de carácter ecológico, social e histórico que la han favorecido. Por este motivo, la caza en Extremadura se caracteriza en el contexto actual por la existencia de un gran número de cazadores, amplia distribución de especies de caza mayor y menor y una superficie acotada que afecta a extensas franjas del territorio. Bajo estas condiciones, la caza en los espacios protegidos de Extremadura se ha visto afectada por una normativa, que si bien fue muy restrictiva en primera instancia, en determinados tipos de espacios, luego ha ido abriéndose tal y como se ha visto reflejado en los instrumentos de planificación, ordenación, gestión y manejo (PRUG PORN) que han sido analizados. La prohibición de la caza en los Parques Naturales no solo produjo conocidos efectos negativos directos (daños a la flora por el crecimiento de poblaciones de determinadas especies, propagación de enfermedades) sino que además evidenció que, ante la ausencia de predadores naturales, no se puede ignorar la necesidad de hacer un control efectivo sobre las poblaciones de determinadas especies. Este control se puede llevar a cabo mediante acciones de caza controladas, con los consiguientes beneficios económicos que se derivarían de tales acciones, o a través de la guardería, en cuyo caso habría que asumir los consiguientes costes, sobre cuya cuantía ya han sido realizados algunos trabajos (Federación Española de Caza, 2012).

En síntesis, la historia es testigo de que caza y conservación han sido, en muchas ocasiones, buenos aliados en diferentes escenarios geográficos. La protección de las especies de caza ha conllevado la protección de los hábitats, como de hecho se pretendía con la constitución de las reservas de caza, beneficiando directa o indirectamente a otras especies. Los espacios protegidos del siglo XXI se extienden por zonas en las que los intereses cinegéticos han sido prioritarios. Por tanto, en territorios como el extremeño, donde la superficie protegida es muy significativa, y además existe una alta coincidencia entre cazaderos tradicionales y espacios naturales protegidos, los diferentes instrumentos legales deben seguir contemplando estas circunstancias. De momento, de los resultados obtenidos en este trabajo se colige que existen restricciones que limitan la práctica de la caza en determinados tipos de espacios de caza, y bajo determinadas circunstancias. Sin embargo, no se puede afirmar que existan medidas excesivamente restrictivas en lo que al aprovechamiento cinegético se refiere en los espacios protegidos de Extremadura, si exceptuamos el ejemplo de los Parques Nacionales.

\section{REFERENCIAS}

Baker, J. E. (1997). Trophy hunting as a sustainable use of wildlife resources in southern and eastern Africa. Journal of sustainable tourism, (5), 306-321. Doi: http://dx.doi.org/10.1080/09669589708667294

Bernabéu Cañete, R. (2002). La caza en Castilla La Mancha y sus estrategias de desarrollo. Cuenca: Ediciones de Universidad de Castilla la Mancha.

Borrell, M.R. (1964). Caza y turismo. Estudios Turísticos, (2), 57-76.

Caspary, H-U. (2001). Regional Dynamics of Hunting and Bushmeat. Utilization in West Africa - An Overview. In M. Bakarr, G. Fonseca, R. Mittermeier, A. Rylands \& K.W. Painemilla (Eds.), Hunting and bushmeat utilization in the African rain forest. Perspectives toward a blue print for conservation action. (pp. 11-16). Washington DC: Conservation International.

Comisión Europea (2008). Documento orientativo sobre la caza de conformidad con la Directiva 79/409/CEE del Consejo relativa a la conservación de las aves silvestres. Directiva de Aves Silvestres. Recuperado de http://ec.europa.eu/environment/nature/conservation/wildbirds/hunting/docs/hunting_guide_es.pdf 
Convention on Biological Diversity (2003). Áreas protegidas: resultados del quinto congreso mundial de parques. Recuperado de http://www.cbd.int/doc/

Convention on Biological Diversity (2004). Addis Ababa principles and guidelines for the sustainable use of biodiversity. Recuperado de https://www.cbd.int/doc/publications/addis-gdl-en.pdf

Council of Europe (2007). European charter on hunting and biodiversity. Recuperado de https://wcd. coe.int/com.instranet.InstraServlet? command=com.instranet. $C$ mdBlobGet \&InstranetImage $=1883$ $\underline{368 \& \text { SecMode }=1 \& \text { DocId }=1436274 \& \text { Usage }=2}$

Europarc (2014). Anuario 2013 de las áreas protegidas de España.

European Environment Agency (2012). Protected areas in Europe, an overview. Recuperado de http://www. eea.europa.eu/publications/protected-areas-in-europe-2012

Federación Española de Caza (2012). Caza, espacios protegidos y desarrollo rural. Conclusiones. Recuperado de http://www.fecaza.com/observatorio/Conclusiones-observatorio-vi.pdf

Fernández, A. (2005). La red de espacios naturales protegidos y la Red natura 2000 en Extremadura. En J.M. López, (ed.): Conservación de la naturaleza en Extremadura (pp. 11-22). Mérida: Junta de Extremadura.

Herruzo, A.C. y Martínez-Jauregui, M. (2013). Trends in hunters, hunting grounds and big game harvest in Spain. Forest System, volumen (22), No 1, 114-122. Doi: http://dx.doi.org/10.5424/fs/2013221-03371

Jones, T. B. (2009). Community benefits from safari hunting and related activities in southern Africa. In B. Dickson, J. Hutton, W.M. Adams (eds.), Recreational hunting, conservation and rural livelihoods (157-177). UK: Wiley Blackwell.

Junta de Extremadura (2014). Informe ambiental de Extremadura2013. Recuperado de http://extremambiente. gobex.es $/$ index.php?view=article\&catid=40\%3Abiblioteca-digital $\&$ id $=4218 \% 3$ Ainforme-ambientalde-extremadura-2013\&option=com_ content\&Itemid=373

Junta de Extremadura (2015). Plan General de Caza. Recuperado de http://extremambiente.gobex.es/files/ Informacion\%20Publica/2015/octbre/Anteproyecto\%20PGCEx\%20-\%20optimizado.pdf

Leader-Williams, N. (2009). Conservation and hunting: friends or foes? En B. Dickson, J. Hutton \& W.M. Adams (Eds.), Recreational hunting, conservation and rural livelihoods (pp. 9-24). Doi: http://dx.doi. org/10.1002/9781444303179.chl

López Ontiveros, A. (1991a). Algunos aspectos de la evolución de la caza en España. Agricultura y Sociedad, (58), 13-51.

López Ontiveros, A. (1991b). La investigación sobre la actividad cinegética en España: estado de la cuestión”. VI Coloquio de Geografía Rural (pp. 145-188). Madrid: Asociación de Geógrafos Españoles.

López Ontiveros, A. (1994). Caza, actividad agraria y geografía en España. Documents d’Análisi Geográfica, número (24), 11-130. Recuperado de http://www.raco.cat/index.php/DocumentsAnalisi/article/ view/41616/5244

López Ontiveros, A. y García Verdugo, FJ. (1991). Geografía de la caza en España. Agricultura y Sociedad, (58), 81-112.

López Ontiveros, A. y Mulero Mendigorri, A. (1998). Recreación rural y caza en España. La investigación hispano-británica reciente en Geografía Rural: del campo tradicional a la transición postproductivista (pp. 215-227). Murcia: Asociación de Geógrafos Españoles.

Magrama (2013). Informe 2011 sobre el Estado del Patrimonio Natural y de la Biodiversidad en España. Recuperado de http://www.magrama.gob.es/es/biodiversidad/temas/inventarios-nacionales/ IEPNB_2011_tcm7-264661.pdf

Martínez de Espinar, A. (1644). Arte de Ballestería y montería. Madrid: Imprenta Real.

Martínez Garrido, E. (2009). Visiones territoriales del boom cinegético español, 1970-1989. Boletín de la A.G.E., (51), 325-351. Recuperado de http://www.age-geografia.es/ojs/index.php/bage/article/ viewFile/1143/1066

Mártínez E., Sánchez, J. y Torija R. (2010). Caza y custodia del territorio en los paisajes agrarios españoles. Actas del XV Coloquio de geografía Rural, (pp. 249-260). Universidad de Extremadura. 
Martínez-Jauregui M., Arenas C. y Herruzo A. C. (2011). Understanding long-term hunting statistics: the case of Spain (1972-2007). Forest Systems, volumen (20 -1), 139-150. Doi: http://dx.doi.org/10.5424/ fs/2011201-10394

Medem Sanjuan, R. (2002). Tras la estrella más alta. Madrid: Agualarga editores.

Mulero Mendigorri A. (1991). Turismo y caza en España. Estado de la cuestión. Agricultura y Sociedad, (58), 147-171.

Mulero Mendigorri, A. (2013). El paisaje forestal-cinegético en Sierra Morena: una lectura geográfica. Cuadernos geográficos, 52 (1), 108-128. Recuperado de http://revistaseug.ugr.es/index.php/cuadgeo/ article/view/928/1079

Muñoz Goyanes, G. (1964). Caza, pesca y parques nacionales. En X ponencia de la primera asamblea nacional de turismo (1-75). Madrid: Ministerio de Información y Turismo.

Muñoz Santos M. y Benayas del Álamo J. (2012). El uso público en la red de Parques Nacionales. Una propuesta de evaluación. Madrid: Organismo Autónomo de parques Nacionales.

OAPN (2013). Memoria de la Red de Parques Nacionales 2013. Recuperado de http://www.magrama.gob.es/ es/red-parques-nacionales/la-red/gestion/memoria 2013 tcm7-361573.pdf

Rengifo Gallego, J. I. (2009). La oferta de caza en España en el contexto del turismo cinegético internacional: las especies de caza mayor. Ería, volumen (78-79), 53-68. Doi: http://dx.doi.org/10.17811/er.0.2009.53-68

Rengifo Gallego, J. I. (2012). Evaluación de la actividad cinegética en Extremadura en los albores del siglo XXI. Retos a corto y medio plazo. Estudios Geográficos, 272, 189-214. Doi: http://dx.doi.org/10.3989/ ESTGEOGR.201207

Tolón Becerra, A. y Lastra Bravo, X. (2008). Los espacios naturales protegidos. Concepto, evolución y situación actual en España. Revista electrónica de Medio Ambiente, 5, 1-25. Recuperado de https:// revistas.ucm.es/index.php/MARE/article/view/MARE0808330001A/15121 